\title{
Can sterile neutrinos be the dark matter?
}

\author{
Uroš Seljak ${ }^{1,2}$, Alexey Makarov ${ }^{1}$, Patrick McDonald ${ }^{3}$, Hy Trac ${ }^{4,1}$ \\ 1 Physics Department, Princeton University, Princeton, NJ 08544, USA; ${ }^{2}$ International Center for Theoretical Physics, \\ Trieste, Italy; ${ }^{3}$ Canadian Institute for Theoretical Astrophysics, University of Toronto, \\ ON M5S 3H8, Canada; ${ }^{4}$ Princeton University Observatory, Princeton, NJ 08544, USA;
}

(Dated: October 29, 2018)

\begin{abstract}
We use the Ly- $\alpha$ forest power spectrum measured by the Sloan Digital Sky Survey (SDSS) and high-resolution spectroscopy observations in combination with cosmic microwave background and galaxy clustering constraints to place limits on a sterile neutrino as a dark matter candidate in the warm dark matter (WDM) scenario. Such a neutrino would be created in the early universe through mixing with an active neutrino and would suppress structure on scales smaller than its free streaming scale. We ran a series of high-resolution hydrodynamic simulations with varying neutrino mass to describe the effect of a sterile neutrino on the Ly- $\alpha$ forest power spectrum. We find that the mass limit is $m_{s}>14 \mathrm{keV}$ at $95 \%$ c.l. (10keV at $99.9 \%$ ), which is nearly an order of magnitude tighter constraint than previously published limits and is above the upper limit allowed by X-ray constraints, excluding this candidate as dark matter in this model. The corresponding limit for a neutrino that decoupled early while in thermal equilibrium is $2.5 \mathrm{keV}$ (95\% c.l.).
\end{abstract}

PACS numbers: 98.80.Jk, 98.80.Cq

One of the major unsolved mysteries in cosmology is the nature of the dark matter in the universe. Observational evidence points towards cold dark matter (CDM), for which random velocities are negligible. Two of the leading particle physics candidates, the lightest supersymmetric partner and axions, both require extensions beyond the standard model. At the same time, neutrino experiments over the past decade have shown that neutrinos oscillate from one flavor to another, which is only possible if they have mass. Current data from atmospheric and solar neutrino experiments [1, 2] are compatible with mixing between the three active neutrino families. Perhaps the simplest way to incorporate these neutrino phenomena into the standard model is to add right-handed neutrinos, just as for other fermions.

Given this extension of the standard model it is natural to ask if these (almost) sterile right-handed neutrinos can also explain the dark matter [3]. At least two sterile neutrinos are required to explain the origin of neutrino mass and existence of different mass mixing scales in solar and atmospheric neutrinos, so in a model with three families of sterile neutrinos a third one can act as dark matter [4]. Such neutrinos free stream and erase all fluctuations on scales smaller than the free streaming length. This length is roughly proportional to the temperature and inversely proportional to the mass of neutrinos. Thus if the neutrino mass is sufficiently high, or the temperature sufficiently low, then it acts just like CDM and can satisfy all of the observational constraints from structure formation. Current constraints require the neutrino mass to be above $1.8 \mathrm{keV}$ [5, 6]. This is below the $5-8 \mathrm{keV}$ upper limits from the absence of detection of X-ray photons from radiative decays $7,[8,9,10]$. A massive neutrino in the $\mathrm{keV}$ range has also been suggested as a possible explanation for high pulsar velocities [11] and such a model can possibly explain baryon asymmetry in the universe 12.
A sterile neutrino is not completely sterile if it is to provide the origin of mass for active neutrinos: it interacts with active neutrinos and the interaction strength is parametrized by the active-sterile mixing angle $\Theta$, which in this model is required to be very small, $\Theta<10^{-4}$. In this regime sterile neutrinos never reach thermal equilibrium [3. In general a sterile neutrino decays into active ones, but the lifetime can be well above the age of the universe over a broad range of masses and mixing angles of interest, so it is effectively stable. If the interaction rate is energy independent then the momentum distribution of sterile neutrinos is simply a reduced version of the distribution of active neutrinos 3]. In practice the interaction rate is not constant over the range of masses of interest, because at temperatures above the QCD transition more interaction channels become available 8 , 13, 14]. In this paper we use the latest calculation [8], which however has only a minor effect relative to the constant interaction rate, reducing the derived mass limits by about $10 \%$ 31].

For $\mathrm{keV}$ masses of interest the corresponding freestreaming length is of order a Megaparsec (Mpc) and below. Distinguishing between cold and warm dark matter thus requires a sensitive probe of linear fluctuations on small scales, but nonlinear evolution erases the initial conditions on these scales today. Of the current tracers of density fluctuations the one that is most suitable for WDM is the Ly- $\alpha$ forest [15]. It is measured from the absorption observed in quasar spectra by neutral hydrogen in the intergalactic medium and has been shown to accurately trace the dark matter distribution [16]. It probes fluctuations down to sub-Mpc scales at redshifts between 2 and 4, so nonlinear evolution, while not negligible, has not erased all of the primordial information. Current WDM constraints from the Ly $\alpha$ forest [5] do not include the latest measurements of the $\operatorname{Ly} \alpha$ forest from the Sloan Digital Sky Survey (SDSS) 17] 32]. The goal 
of this letter is to derive new limits by incorporating these observational constraints and combining them with a series of new hydrodynamic simulations which accurately describe the effect of a massive neutrino on the Ly- $\alpha$ forest.

The linear theory calculations of WDM using CMBFAST [18] result in the matter power spectra shown, relative to $\mathrm{CDM}$, in the upper left panel of figure 1 $\mathrm{We}$ plot the ratio of WDM to CDM power for $m_{\nu}=6.5,10$, 14 and $20 \mathrm{keV}$. Instead of the usual $3 \mathrm{~d}$ power spectrum we plot the corresponding $1 \mathrm{~d}$ projection, which is more relevant for the comparison to the $1 \mathrm{~d}$ Ly $\alpha$ forest observations. One can see the suppression of power on scales smaller than the free-streaming length, which depends on the neutrino mass. While for $m_{\nu}>10 \mathrm{keV}$ there is hardly any effect for $k<5 \mathrm{~h} / \mathrm{Mpc}$ in $3 \mathrm{~d}$ (see e.g. relevant figures in [5, [6]), the corresponding $1 \mathrm{~d}$ power spectrum shows more of an effect because small scale modes in $3 \mathrm{~d}$ are projected to large scale modes in $1 \mathrm{~d}$. For example, for $m_{\nu}=20 \mathrm{keV}$ there is essentially no effect in $3 \mathrm{~d}$ for $k<3 \mathrm{~h} / \mathrm{Mpc}$ and even at $k=5 \mathrm{~h} / \mathrm{Mpc}$ the power suppression is only $2 \%$. SDSS measurements of the flux power in $1 \mathrm{~d}$ do not extend above $2 \mathrm{~h} / \mathrm{Mpc}$ and high resolution spectra are reliable up to $5 \mathrm{~h} / \mathrm{Mpc}$. So if one were interpreting them as measuring $3 \mathrm{~d}$ power then it would be very difficult to detect neutrino masses in this mass range. However, the corresponding $1 \mathrm{~d}$ case in figure 1 shows a $3 \%$ power suppression at $2 \mathrm{~h} / \mathrm{Mpc}$ and $15 \%$ at $5 \mathrm{~h} / \mathrm{Mpc}$. This rapidly increases with declining mass, so that for a $6.5 \mathrm{keV}$ neutrino the power suppression is $15 \%$ at $\mathrm{k}=2 \mathrm{~h} / \mathrm{Mpc}$ and a factor of 2 at $\mathrm{k}=5 \mathrm{~h} / \mathrm{Mpc}$.

Nonlinear evolution and hydrodynamic effects further modify the linear predictions, which must be addressed with simulations. We ran hydrodynamic simulations for a series of neutrino masses ranging from $3.4 \mathrm{keV}$ to $20 \mathrm{keV}$. Many convergence tests and comparisons between different hydrodynamic codes have been performed, which will be presented in a separate publication. These tests confirm the accuracy of the original analysis in [19], which was based on a grid of hydro-PM simulations sparsely calibrated with hydrodynamic simulations. For the hydrodynamic simulations in this paper we used the Eulerian moving frame TVD+PM code described in 20]. The Eulerian conservation equations are solved in a frame moving with the fluid where numerical Mach numbers are minimized, allowing thermodynamic variables to be accurately calculated for both subsonic and supersonic gas. Our standard simulations used $20 \mathrm{Mpc} / \mathrm{h}$ boxes with $256^{3}$ particles for dark matter and $512^{3}$ cells for gas. We used $10 \mathrm{Mpc} / \mathrm{h}$ boxes with equal or twice this resolution to test convergence.

Simulation results are shown in figure 1 for redshifts 2, 3 and 4 that span the observational range. We have adjusted the level of the UV background to match the mean absorption as measured from the data. The results show that for $m_{\nu}=20 \mathrm{keV}$ there are 1-2 percent effects at $\mathrm{k}=2 \mathrm{~h} / \mathrm{Mpc}$ at $\mathrm{z}=4$, increasing to $11 \%$ for $m_{\nu}=6.5 \mathrm{keV}$. At $\mathrm{k}=5 \mathrm{~h} / \mathrm{Mpc}$ the effects are $6 \%$ sup-

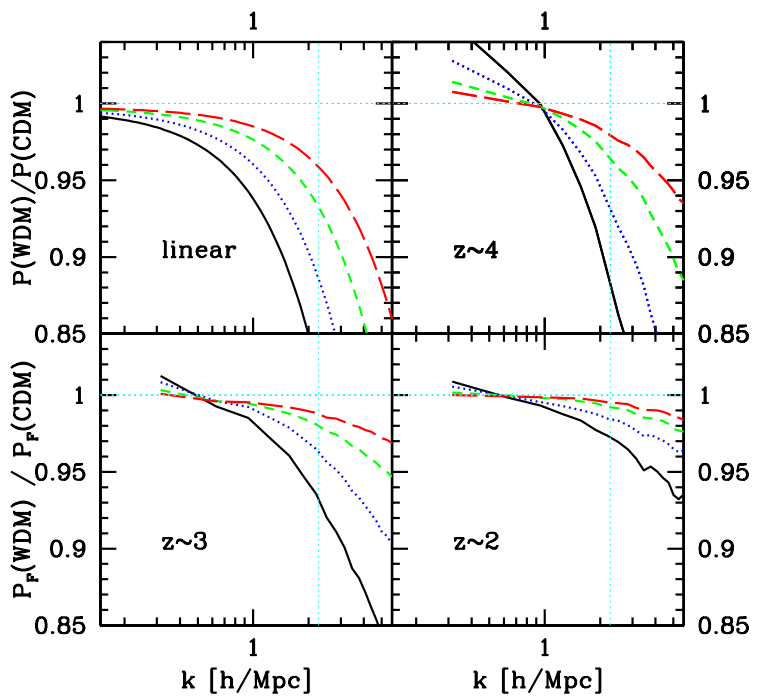

FIG. 1: Ratio of WDM power spectrum relative to CDM shown over the relevant observational range. From left to right the sterile neutrino masses are $6.5 \mathrm{keV}, 10 \mathrm{keV}, 14 \mathrm{keV}$ and $20 \mathrm{keV}$. Top left corner shows $1 \mathrm{~d}$ linear power spectrum, while the other 3 panels show the ratios from hydrodynamic simulations at redshifts 2,3 and 4 . We used concordance cosmology with $\Omega_{m}=0.28$ and $H_{0}=71 \mathrm{~km} / \mathrm{s} / \mathrm{Mpc}$. Dashed vertical line shows the upper limit on $k$ for SDSS data.

pression for $m_{\nu}=20 \mathrm{keV}$ mass and a factor of 1.5 for $m_{\nu}=6.5 \mathrm{keV}$. These are redshift dependent: while there is little differentiating power between models at low redshift, the differences become significantly larger at high redshift, where the mean level of absorption is higher and the linear power is better preserved. Finally, we note that the suppression of small scale power also affects the large scale bias of the flux power spectrum, which explains why the ratios do not converge to unity on large scales.

Since the free-streaming scale for these high mass neutrinos is so small one must be careful to have sufficient spatial resolution to capture the suppression of power on small scales. This is not a trivial requirement: for a $10 \mathrm{keV}$ neutrino the scale corresponding to a $50 \%$ suppression of the WDM transfer function relative to CDM occurs at 4 times the cell size in our standard simulations (1/4 of the Nyquist frequency - for a $20 \mathrm{keV}$ neutrino the corresponding number is $1 / 2$ ). Doubling the resolution does not change our results significantly $(<20 \%$ of the size of the WDM effect), so our simulations have converged over the relevant mass range, but only barely. It is important to note that insufficient resolution weakens the constraints, since the power suppression is not captured on scales below the Nyquist scale corresponding to the cell size. This could be an issue for the SPH simulations used in [5]: for example, for their $10 \mathrm{keV}$ sterile neutrino mass simulation (corresponding to $2 \mathrm{keV}$ thermal neutrino in their plots) they find essentially no effect in the Ly $\alpha$ forest on the observable scales, concluding 
that such masses cannot be probed by the Ly $\alpha$ forest, in clear contradiction with our results in figure 1 In their simulations a $50 \%$ suppression of the $10 \mathrm{keV}$ WDM transfer function relative to CDM happens at the scale of the particle separation. This is a factor of 4 worse resolution than our cell size for gas, and a factor of 2 worse than our particle spacing for dark matter. Thus much of the power suppression is missing already in the initial conditions and the Lagrangian spatial resolution in the SPH simulations cannot restore it (additionally, it is unclear that the Lagrangian nature of the SPH should help for the near-mean or even underdense gas probed by the $\operatorname{Ly} \alpha$ forest at $z \sim 4$ ).

In addition to the Ly- $\alpha$ forest flux power spectrum from SDSS [17] we have added earlier high-resolution Ly $\alpha$ forest constraints in a weak form 21, 22]. When testing the robustness of the derived constraints we also include the more recent high-resolution Ly $\alpha$ forest data [23, 24]. While galaxy clustering and CMB data do not constrain WDM, they are useful for constraining the remaining cosmological parameters. We use as inputs the SDSS galaxy power spectrum 25 and CMB power spectrum from WMAP [26]. Our analysis is based on the Monte Carlo Markov Chain (MCMC) method [27] and uses CMBFAST [18] to output both CMB spectra and the corresponding matter power spectra $P(k)$. The output transfer functions are interpolated onto a grid of simulations using the matter power spectra rather than the neutrino mass, since it is the matter spectrum that is most directly related to the observations.

Our most general cosmological parameter space has 9 parameters, which are the Hubble constant, matter and baryon density, amplitude, slope and running of the primordial power spectrum, tensor to scalar ratio, optical depth and neutrino mass. Since in most models of inflation tensors and running are expected to be small we also explore the constraints when they are set to zero.

We compare the theoretical $P_{F}(k)$ directly to the measured power spectrum. This is particularly important for the WDM analysis, where one cannot use the $3 \mathrm{~d}$ linear power spectrum amplitude and slope constraints as given in [19], since as emphasized there these are valid only in the context of standard CDM models without WDM. The Ly $\alpha$ forest contains several nuisance parameters which we are not interested in for the cosmological analysis, so they are marginalized over. These include the UV background intensity, temperature-density relation of the gas and the filtering length (related to Jeans scale [28]). We also include a marginalization over several additional physical effects, such as fluctuations in the UV background and galactic winds [19, 29].

Applying the standard MCMC analysis to the WDM case we find no evidence of WDM: the limit is $m_{\nu}>$ $14 \mathrm{keV}$ at $95 \%$ c.l. ( $10 \mathrm{keV}$ at $99.9 \%$ c.l.). The corresponding limit for neutrinos which were in thermal equilibrium at a high temperature, when the universe had more degrees of freedom, and then decoupled, is $m_{\nu}<2.5 \mathrm{keV}$ at $95 \%$ c.l. This constraint is obtained

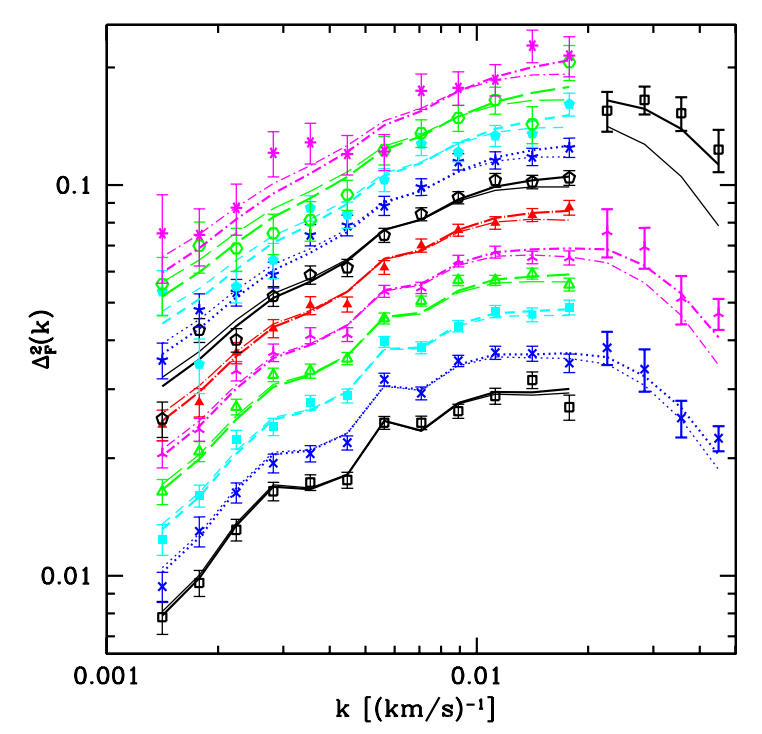

FIG. 2: To the left are the observed SDSS Ly $\alpha$ forest flux power spectra as a function of redshift from 2.2 (bottom) to 4.2 (top) in steps of 0.2 . To the right are the power spectra from the high resolution data compiled at redshifts 2.4, 3.0 and 3.9. For each redshift the thick lines are from the best fitted CDM model, while the (generally lower at high $\mathrm{k}$ ) thin lines are for the corresponding WDM model with $6.5 \mathrm{keV}$ sterile neutrino. The latter is discrepant with both SDSS and high resolution data, with most of the distinguishing power coming from higher redshifts.

in our 9 parameter space, but reducing the parameter space to the minimal 7 parameters without running and tensors does not change the results. The Ly $\alpha$ forest data, best fitted CDM model and corresponding WDM model for $m_{\nu}=6.5 \mathrm{keV}$ are shown in figure 2 One can see how the suppression of power on small scales in WDM makes the fit worse. For this figure, where we have not adjusted all the other parameters to their best fitted value, the increase in $\chi^{2}$ with WDM is 77 - when the Ly $\alpha$ forest model parameters and power spectrum amplitude and slope are fitted, $\Delta \chi^{2}$ is still 27 . Even without high resolution constraints, the poor fit to the SDSS data is apparent, especially at higher redshifts. Removing the high resolution data only weakens the bounds by $15 \%$. The converse however is not true: without SDSS the previously found constraint (after 10\% adjustment for nonthermal momentum distribution) is $m_{\nu}>1.8 \mathrm{keV}$ (95\% c.l.) [5]. This is because within the high resolution data there are degeneracies between WDM and many of the nuisance parameters such as the temperature of the IGM, UV flux and filtering scale. These can be removed by adding the large scale flux power spectrum measured by the SDSS data. Finally, we note that using the more recent highresolution Ly $\alpha$ forest data 23, 24] does not improve the limits obtained above.

Sterile neutrinos that couple to active ones also decay and their radiative decays result in photons with energy 
peaking at close to one half of the neutrino mass, which for $\mathrm{keV}$ masses can be searched for in X-rays from either clusters or from their cumulative contribution in a random direction. Absence of such X-ray emission in the Virgo cluster results in an upper limit on the mass of $8 \mathrm{keV}$ [8] while recent reevaluation of X-ray background constraints gives an upper limit of $5 \mathrm{keV}$ for the value of mixing angle that matches the required density of sterile neutrinos [10]. These are all below our 99.9\% lower limit, suggesting sterile neutrinos cannot be the dark matter in this model.

Can the bounds presented here be invalidated by some additional physical effect in Ly $\alpha$ forest that is not included in our model? This is unlikely, but cannot be ruled out completely. There are possible physical effects that can in principle affect the Ly $\alpha$ forest power spectrum and while most of them have been shown to be negligible or are already part of our standard analysis [29, 30], there remains a possibility that something else will turn out to be important. However, it is important to recognize how successful is the current model in explaining the observations. Any potential effects that may be missing in the current analysis are constrained by the remarkable agreement of the simplest CDM model with the data. It seems unlikely that if we lived in a WDM universe its signature were erased exactly by some (yet to be discovered) physical effect. Barring any such cancellations we may conclude that the simplest model of sterile neutrinos as the dark matter is ruled out, since the upper limit from their decays and the lower limit from their effect on large scale structure no longer leaves an open window.

U.S. is supported by the Packard Foundation, NASA NAG5-1993 and NSF CAREER-0132953. H.T. acknowledges NASA grant NNG04GC50G. We thank Alexei Smirnov for useful discussions.
[1] Y. Ashie, J. Hosaka, K. Ishihara, Y. Itow, J. Kameda, Y. Koshio, A. Minamino, C. Mitsuda, M. Miura, S. Moriyama, et al., Physical Review Letters 93, 101801 (2004).

[2] Q. R. Ahmad, R. C. Allen, T. C. Andersen, J. D. Anglin, G. Bühler, J. C. Barton, E. W. Beier, M. Bercovitch, J. Bigu, S. Biller, et al., Physical Review Letters 87, 071301 (2001).

[3] S. Dodelson and L. M. Widrow, Physical Review Letters 72, 17 (1994).

[4] T. Asaka, S. Blanchet, and M. Shaposhnikov, Phys. Lett. B631, 151 (2005), hep-ph/0503065.

[5] M. Viel, J. Lesgourgues, M. G. Haehnelt, S. Matarrese, and A. Riotto, Phys. Rev. D 71, 063534 (2005).

[6] K. Abazajian, ArXiv Astrophysics e-prints (2005), arXiv:astro-ph/0512631.

[7] K. Abazajian, G. M. Fuller, and W. H. Tucker, Astrophys. J. 562, 593 (2001).

[8] K. Abazajian, ArXiv Astrophysics e-prints (2005), arXiv:astro-ph/0511630.

[9] M. Mapelli and A. Ferrara, Mon. Not. R. Astron. Soc. 364, 2 (2005).

[10] A. Boyarsky, A. Neronov, O. Ruchayskiy, and M. Shaposhnikov, ArXiv High Energy Physics - Phenomenology e-prints (2006), arXiv:hep-ph/0601098.

[11] A. Kusenko and G. Segrè, Physics Letters B 396, 197 (1997).

[12] T. Asaka and M. Shaposhnikov, Phys. Lett. B620, 17 (2005), hep-ph/0505013.

[13] A. D. Dolgov and S. H. Hansen, Astroparticle Physics 16, 339 (2002).

[14] K. Abazajian, G. M. Fuller, and M. Patel, Phys. Rev. D 64, 023501 (2001).

[15] V. K. Narayanan, D. N. Spergel, R. Davé, and C. Ma, Astrophys. J. Lett. 543, L103 (2000).

[16] R. A. C. Croft, D. H. Weinberg, N. Katz, and L. Hernquist, Astrophys. J. 495, 44 (1998).

[17] P. McDonald, U. Seljak, S. Burles, D. J. Schlegel, D. H. Weinberg, D. Shih, J. Schaye, D. P. Schneider, J. Brinkmann, R. J. Brunner, et al., ArXiv Astrophysics e-prints (2004), astro-ph/0405013.

[18] U. Seljak and M. Zaldarriaga, Astrophys. J. 469, 437 (1996).

[19] P. McDonald, U. Seljak, R. Cen, D. Shih, D. H. Weinberg, S. Burles, D. P. Schneider, D. J. Schlegel, N. A. Bahcall, J. W. Briggs, et al., Astrophys. J. 635, 761 (2005).

[20] H. Trac and U. Pen, New Astronomy 9, 443 (2004).

[21] P. McDonald, J. Miralda-Escudé, M. Rauch, W. L. W. Sargent, T. A. Barlow, R. Cen, and J. P. Ostriker, Astrophys. J. 543, 1 (2000).

[22] P. McDonald, J. Miralda-Escudé, M. Rauch, W. L. W. Sargent, T. A. Barlow, and R. Cen, Astrophys. J. 562, $52(2001)$.

[23] R. A. C. Croft, D. H. Weinberg, M. Bolte, S. Burles, L. Hernquist, N. Katz, D. Kirkman, and D. Tytler, Astrophys. J. 581, 20 (2002).

[24] T. . Kim, M. Viel, M. G. Haehnelt, R. F. Carswell, and S. Cristiani (2003), astro-ph/0308103.

[25] M. Tegmark, M. R. Blanton, M. A. Strauss, F. Hoyle, D. Schlegel, R. Scoccimarro, M. S. Vogeley, D. H. Weinberg, I. Zehavi, A. Berlind, et al., Astrophys. J. 606, 702 (2004).

[26] G. Hinshaw, D. N. Spergel, L. Verde, R. S. Hill, S. S. Meyer, C. Barnes, C. L. Bennett, M. Halpern, N. Jarosik, A. Kogut, et al., Astrophys. J. Supp. 148, 135 (2003).

[27] U. Seljak, P. McDonald, and A. Makarov, Mon. Not. R. Astron. Soc. 342, L79 (2003).

[28] N. Y. Gnedin and L. Hui, Mon. Not. R. Astron. Soc. 296, 44 (1998).

[29] P. McDonald, U. Seljak, R. Cen, P. Bode, and J. P. Ostriker, Mon. Not. R. Astron. Soc. 360, 1471 (2005).

[30] K. Lai, A. Lidz, L. Hernquist, and M. Zaldarriaga, ArXiv Astrophysics e-prints (2005), arXiv:astro-ph/0510841.

[31] We assume throughout the paper a negligible lepton asymmetry and do not consider the possibility of a resonant enhancement in neutrino production, nor do we consider the possibility for additional creation processes of the sterile neutrino, such as the coupling to inflaton.

[32] The exception is [6], where however the linear power spec- 
trum determination of [19], only valid for CDM, was applied to WDM models, making the results unreliable. 